\title{
ON BOUNDEDLY COMPLETE TRANSFINITE BASES IN BANACH SPACES
}

\author{
P.K. JAIN AND S.K. KAUSHIK
}

\section{Introduction}

Initially, Bessaga [1] defined the concept of monotone transfinite basis which, in fact he called 'monotone basis of type $\nu$ '. Subsequently, Bessaga [2] replaced the condition of monotonicity by a weaker condition of uniform boundedness and introduced the term 'projection basis of type $\nu$ '. However, the definition of the transfinite basis, we use in the present note is due to Dorembus [5] who, in fact, worked directly with transfinite bases of subspaces.

In the present note we consider two properties, (I) and (II), in Banach spaces having transfinite bases and observe that they are equivalent. We also prove that if a transfinite basis satisfies (II) (or (I)), then the transfinite basis is boundedly complete in the sense of Bessaga [3] and an example has been exhibited to show that the converse need not be true. This paper may be regarded in a sequel to [6].

\section{Preliminaries and lemmas}

Throughout $E$ will denote a Banach space over the scalar field $\mathbb{K}(\mathbb{R}$ or $\mathbb{C}), \nu$ an infinite ordinal and $\left[x_{\lambda}\right]_{\lambda<\nu}$ the closed linear span of $\left\{x_{\lambda}\right\}_{\lambda<\nu}$.

For the terms like transfinite biorthogonal system, associated transfinite sequence of coefficient functionals (a.t.s.c.f), convergence of a transfinite series, transfinite basis, boundedly complete transfinite basis, sequence of partial sum operators $\left\{S_{\lambda}\right\}_{\lambda<\nu}$ and uniformly convex Banach space, one may refer to [7].

Lemma [6]. Let $\left\{x_{\lambda}\right\}_{\lambda<\nu}$ be a transfinite sequence of non-zero elements in $E$ such that $\left[x_{\lambda}\right]_{\lambda<\nu}=E$. Then $\left\{x_{\lambda}\right\}_{\lambda<\nu}$ is a transfinite basis in $E$ if and only if there exists $a$ constant $M \geq 1$ such that

$$
\left\|\sum_{\chi<\lambda} \alpha_{\chi} x_{\chi}\right\| \leq M\left\|\sum_{\chi<\mu} \alpha_{\chi} x_{\chi}\right\|
$$

for all ordinals $\lambda, \mu$ with $\lambda \leq \mu<\nu$ and arbitrary scalars $\alpha_{1}, \alpha_{2}, \ldots, \alpha_{\mu}$ in $\mathbb{K}$ such that $\sum_{\chi<\mu} \alpha_{\chi} x_{\chi}$ converges.

Received February 22, 1988; revised March 9, 1989.

Subject Classification 1980 (Revision 1985): AMO(MOS)46B15.

The research of the author is supported by the CSIR (India). 
The infimum of $M$, denoted by $V_{\left\{x_{\lambda}\right\}_{\lambda<\nu}}$, is called the norm of the transfinite basis $\left\{x_{\lambda}\right\}_{\lambda<\nu}$. In case, $V_{\left\{x_{\lambda}\right\}_{\lambda<\nu}}=1$, the transfinite basis $\left\{x_{\lambda}\right\}_{\lambda<\nu}$ is called monotone.

\section{Main results}

Let $\left\{x_{\lambda}\right\}_{\lambda<\nu}$ be a transfinite basis in $E$. Then :

(a) $\left\{x_{\lambda}\right\}_{\lambda<\nu}$ is said to satisfy property (I) if for each $c>0$, there exists a number $\gamma_{c}>0$ (independent of $\left\{\alpha_{\lambda}\right\}_{\lambda<\nu}$ and $\mu$ ) such that

$$
\left\|\sum_{\chi \leq \mu} \alpha_{\chi} x_{\chi}\right\|=1,\left\|\sum_{\mu+1 \leq \chi<\nu} \alpha_{\chi} x_{\chi}\right\| \geq c \Rightarrow\left\|\sum_{\chi<\nu} \alpha_{\chi} x_{\chi}\right\| \geq 1+\gamma_{c}
$$

where $\left\{\alpha_{\lambda}\right\}_{\lambda<\nu}$ is any transfinite sequence in $\mathbb{K}$.

(b) $\left\{x_{\lambda}\right\}_{\lambda<\nu}$ is said to satisfy property (II) if for each $\epsilon>0$, there exists a $\delta>0$ (independent of $\left\{\alpha_{\lambda}\right\}_{\lambda<\nu}$ and $\mu$ ) such that

$$
\left\|\sum_{\chi \leq \mu} \alpha_{\chi} x_{\chi}\right\|>1-\delta,\left\|\sum_{\chi<\nu} \alpha_{\chi} x_{\chi}\right\|=1 \Rightarrow\left\|\sum_{\mu+1 \leq \chi<\nu} \alpha_{\chi} x_{\chi}\right\| \leq \epsilon,
$$

where $\left\{\alpha_{\lambda}\right\}_{\lambda<\nu}$ is any transfinite sequence in $\mathbb{K}$ such that $\sum_{\chi<\nu} \alpha_{\chi} x_{\chi}$ converges.

These two properties are equivalent.

\section{Justification.}

(I) $\Rightarrow$ (II). Suppose that the transfinite basis $\left\{x_{\lambda}\right\}_{\lambda<\nu}$ satisfies property (I) but not property (II). Then, there exists an $\epsilon>0$ such that for every $\delta>0$, there is a transfinite sequence of scalars $\left\{\alpha_{\lambda}\right\}_{\lambda<\nu} \subset \mathbb{K}$ with $\sum_{\lambda<\nu} \alpha_{\lambda} x_{\lambda}$ convergent and an ordinal $\mu<\nu$ such that

$$
\left\|\sum_{\chi \leq \mu} \alpha_{\chi} x_{\chi}\right\|>1-\delta,\left\|\sum_{\chi<\nu} \alpha_{\chi} x_{\chi}\right\|=1 \text { but }\left\|\sum_{\mu+1 \leq \chi<\nu} \alpha_{\chi} x_{\chi}\right\|>\epsilon .
$$

Let $\gamma_{c}>0$ be any arbitrary number. Put $\delta=\gamma_{c}\left(1+\gamma_{c}\right)^{-1}$ and $\beta_{\chi}=\alpha_{\chi}\left(\left\|\sum_{\chi \leq \mu} \alpha_{\chi} x_{\chi}\right\|\right)^{-1},(\chi<\nu)$. Then

$$
\left\|\sum_{\chi \leq \mu} \beta_{\chi} x_{\chi}\right\|=1
$$

and

$$
\begin{aligned}
\left\|\sum_{\mu+1 \leq \chi<\nu} \beta_{\chi} x_{\chi}\right\| & >\epsilon\left(\left\|\sum_{\chi \leq \mu} \alpha_{\chi} x_{\chi}\right\|\right)^{-1} \\
& \geq \epsilon / V_{\left\{\chi_{\lambda}\right\}_{\lambda<\nu}}=c>0
\end{aligned}
$$

where $V_{\left\{x_{\lambda}\right\}_{\lambda<\nu}}$ is the norm of the transfinite basis $\left\{x_{\lambda}\right\}_{\lambda<\nu}$. But

$$
\left\|\sum_{\chi<\nu} \beta_{\chi} x_{\chi}\right\|<1+\gamma_{c}
$$


Thus $\left\{x_{\lambda}\right\}_{\lambda<\nu}$ does not satisfy property (I).

(II) $\Rightarrow$ (I). Let $\left\{x_{\lambda}\right\}_{\lambda<\nu}$ satisfy property (II) but not property (I). Then, there exists a $c>0$ such that for every $\gamma_{c}>0$, there is a transfinite sequence of scalars $\left\{\alpha_{\lambda}\right\}_{\lambda<\nu} \subset \mathbb{K}$ and an ordinal $\mu<\nu$ such that

$$
\left\|\sum_{\chi \leq \mu} \alpha_{\chi} x_{\chi}\right\|=1,\left\|\sum_{\mu+1 \leq \chi<\nu} \alpha_{\chi} x_{\chi}\right\| \geq c \text { but }\left\|\sum_{\chi<\nu} \alpha_{\chi} x_{\chi}\right\|<1+\gamma_{c} .
$$

We now show that there is an $\epsilon>0$ such that for no $\delta>0$, the relation in property (II) is satisfied. Let $0<\eta<1$ be arbitrary but fixed. Let $\delta>0$ be arbitrary such that $\delta \leq \eta$. Put $\gamma_{c}=\delta(1-\delta)^{-1}$ and $\beta_{\chi}=\alpha_{\chi}\left(\left\|\sum_{\chi<\nu} \alpha_{\chi} x_{\chi}\right\|\right)^{-1}$.

Then

$$
\left\|\sum_{\chi \leq \mu} \beta_{\chi} x_{\chi}\right\|>1-\delta \quad \text { and } \quad\left\|\sum_{\chi<\nu} \beta_{\chi} x_{\chi}\right\|=1
$$

But

$$
\begin{aligned}
\left\|\sum_{\mu+1 \leq \chi<\nu} \beta_{\chi} x_{\chi}\right\| & \geq c\left(\left\|\sum_{\chi<\nu} \alpha_{\chi} x_{\chi}\right\|\right)^{-1} \\
& >c(1-\delta) \\
& \geq c(1-\eta) .
\end{aligned}
$$

Taking $\epsilon=c(1-\eta)$ our assertion is established.

Theorem 1. If $\left\{x_{\lambda}\right\}_{\lambda<\nu}$ is a monotone transfinite basis in a uniformly convex Banach space $E$, then $\left\{x_{\lambda}\right\}_{\lambda<\nu}$ satisfies property (II) (and hence properiy (I)).

Proof. Suppose on the contrary that $\left\{x_{\lambda}\right\}_{\lambda<\nu}$ does not satisfy property (II). Then, there exists an $\epsilon>0$ such that for every $\delta>0$ one can find $\left\{\alpha_{\lambda}^{(\delta)}\right\}_{\lambda<\nu} \subset \mathbb{K}$ with $\sum_{\lambda<\nu} \alpha_{\lambda}^{(\delta)} x_{\lambda}$ converging in $E$, and an ordinal number $\mu(\delta)<\nu$ such that

$$
\left\|\sum_{\chi \leq \mu(\delta)} \alpha_{\chi}^{(\delta)} x_{\chi}\right\|>1-\delta, \quad\left\|\sum_{\chi<\nu} \alpha_{\chi}^{(\delta)} x_{\chi}\right\|=1
$$

and

$$
\left\|\sum_{\mu(\delta)+1 \leq \chi<\nu} \alpha_{\chi}^{(\delta)} x_{\chi}\right\|>\epsilon
$$

Then, in view of monotonicity of $\left\{x_{\lambda}\right\}_{\lambda<\nu}$, we have

$$
1-\delta<\left\|\sum_{\chi \leq \mu(\delta)} \alpha_{\chi}^{(\delta)} x_{\chi}\right\| \leq\left\|\sum_{\chi<\nu} \alpha_{\chi}^{(\delta)} x_{\chi}\right\|=1 .
$$

Since $E$ is uniformly convex, there is a $\delta_{0} \equiv \delta_{0}(\epsilon), 0<\delta_{0}<1$, such that whenever $\|x\| \leq 1,\|y\| \leq 1$ and $\|x-y\|>\epsilon$, we have $\left\|\frac{x+y}{2}\right\| \leq 1-\delta_{0}$. Writing

$$
x_{0}=\sum_{\chi \leq \mu\left(\delta_{0}\right)} \alpha_{\chi}^{\left(\delta_{0}\right)} x_{\chi} \quad \text { and } \quad y_{0}=\sum_{\chi<\nu} \alpha_{\chi}^{\left(\delta_{0}\right)} x_{\chi},
$$


we have $\left\|x_{0}\right\| \leq 1,\left\|y_{0}\right\|=1$ and $\left\|x_{0}-y_{0}\right\|>\epsilon$. Hence

$$
\begin{aligned}
1-\delta_{0} & \leq\left\|\frac{x_{0}+y_{0}}{2}\right\| \\
& =\left\|\sum_{\chi \leq \mu\left(\delta_{0}\right)} \alpha_{\chi}^{\left(\delta_{0}\right)} x_{\chi}+\frac{1}{2} \sum_{\mu\left(\delta_{0}\right)+1 \leq x<\nu} \alpha_{\chi}^{\left(\delta_{0}\right)} x_{\chi}\right\| \\
& \geq\left\|\sum_{\chi \leq \mu\left(\delta_{0}\right)} \alpha_{\chi}^{\left(\delta_{0}\right)} x_{\chi}\right\|>1-\delta_{0} .
\end{aligned}
$$

This is absurd.

Theorem 2. If $\left\{x_{\lambda}\right\}_{\lambda<\nu} \subset E$ is a transfinite basis satisfying property (I), then $\left\{x_{\lambda}\right\}_{\lambda<\nu}$ is a boundedly complete transfinite basis.

Proof. Let $\lambda_{1}<\lambda_{2}<\ldots<\lambda \leq \nu$ be such that $\lim _{j \rightarrow \infty} \lambda_{j}=\lambda$ and $\left\{y_{n}\right\} \subset E$ be any (countable) bounded sequence such that

$$
S_{\lambda_{n}}\left(y_{j}\right)=y_{n}, \quad(n=1,2, \ldots, j ; j \in N) .
$$

To complete the proof, it is enough to establish that $\lim _{n \rightarrow \infty} y_{n}=x(\in E)$ exists. Choose a sequence $\left\{n_{k}\right\}$ of positive integers such that

$$
\lim _{k \rightarrow \infty}\left\|y_{n_{k}}\right\|=\varlimsup_{n \rightarrow \infty}\left\|y_{n}\right\|=B
$$

If $B=0$, then $\lim _{n \rightarrow \infty} y_{n}=0(\in E)$ exists. If $B \neq 0$, we shall show that $\left\{y_{n_{k}}\right\}$ is a cauchy sequence. Suppose on the contrary that $\left\{y_{n_{k}}\right\}$ is not a cauchy sequence, then there exists a $\delta>0$ and subsequences $\left\{y_{n_{k_{j}}}\right\},\left\{y_{n_{p_{j}}}\right\}$ of $\left\{y_{n_{k}}\right\}$ with $n_{k_{j}}>n_{p_{j}},(j \in N)$ such that

$$
\left\|y_{n_{k_{j}}}-y_{n_{p_{j}}}\right\| \geq \delta, \quad(j \in \mathbb{N}) \text {. }
$$

Since

$$
\left\|\frac{y_{n_{k_{j}}}-y_{n_{p_{j}}}}{\left\|y_{n_{p_{j}}}\right\|}\right\| \geq \frac{\delta}{A}=c>0
$$

where $A=\sup _{n}\left\|y_{n}\right\|<\infty$, it follows, in view of Property (I), that

$$
\lim _{j \rightarrow \infty}\left\|y_{n_{k_{j}}}\right\| \geq \lim _{j \rightarrow \infty}\left\|y_{n_{p_{j}}}\right\|\left(1+\gamma_{c}\right)
$$

This gives $B \geq B\left(1+\gamma_{c}\right)$, which is impossible since $B \neq 0$.

Hence, $\left\{y_{n_{k}}\right\}$ is a cauchy sequence in $E$. Let $\lim _{k \rightarrow \infty} y_{n_{k}}=x$, where $x \in E$. Then

$$
\begin{aligned}
x=\lim _{k \rightarrow \infty} y_{n_{k}} & =\lim _{k \rightarrow \infty} S_{\lambda_{n_{k}}}\left(y_{j}\right) \\
& =S_{\lambda}\left(y_{j}\right) \\
& =\lim _{n \rightarrow \infty} S_{\lambda_{n}}\left(y_{j}\right), \quad(j \in \mathbb{N}) \\
& =\lim _{n \rightarrow \infty} y_{n} .
\end{aligned}
$$


This completes the proof.

Corollary. If $\left\{x_{\lambda}\right\}_{\lambda<\nu}$ is a monotone transfinite basis of a uniformly convex Banach Space $E$, then $\left\{x_{\lambda}\right\}_{\lambda<\nu}$ is boundedly complete transfinite basis of $E$.

The converse of the Theorem 2 is not true.

Example. Let

$$
\begin{aligned}
E & =l^{1}[\nu],[\nu]=[1, \nu) \\
& =\left\{\left\{\alpha_{\chi}\right\}_{\chi<\nu} \subset K \mid \operatorname{card}\left\{\lambda: \alpha_{\lambda} \neq 0\right\} \leq \mathcal{N}_{0}\right\}
\end{aligned}
$$

where $\left\|\left\{\alpha_{\lambda}\right\}_{\lambda<\nu}\right\|=\sum_{\chi<\nu}\left|\alpha_{\chi}\right|<\infty$. Let $\left\{e_{\lambda}\right\}_{\lambda<\nu}$ be the natural transfinite basis of $l^{1}[\nu]$. Define a transfinite sequence $\left\{x_{\lambda}\right\}_{\lambda<\nu}$ in $E$ by $x_{1}=\frac{1}{2}\left(e_{1}+e_{2}\right), x_{2}=\frac{1}{2}\left(-e_{1}+e_{2}\right)$ and $x_{\lambda}=e_{\lambda}$ for $2<\lambda<\nu$. Then, $\left\{x_{\lambda}\right\}_{\lambda<\nu}$ is a transfinite basis for $l^{1}[\nu]$, since for any $x=\left\{\alpha_{\lambda}\right\}_{\lambda<\nu} \in l^{1}[\nu]$, there is a unique transfinite sequence $\left\{\beta_{\lambda}\right\}_{\lambda<\nu}$ given by $\beta_{1}=\alpha_{1}+\alpha_{2}, \beta_{2}=-\alpha_{1}+\alpha_{2}$ and $\beta_{\lambda}=\alpha_{\lambda}$ for $2<\lambda<\nu$, satisfying

$$
x=\sum_{\lambda<\nu} \beta_{\lambda} x_{\lambda} .
$$

Furthermore, $\left\{x_{\lambda}\right\}_{\lambda<\nu}$ is boundedly complete. Indeed, if $\lambda_{1}<\lambda_{2}<\ldots<\lambda \leq \nu$ such that $\lim _{j \rightarrow \infty} \lambda_{j}=\lambda$ and $\left\{y_{n}\right\} \subset E$ is any (countable) bounded sequence satisfying

$$
S_{\lambda_{n}}\left(y_{j}\right)=y_{n}, \quad(n=1,2, \ldots, j ; j \in N),
$$

then

$$
\begin{aligned}
\sum_{\chi<\lambda_{n}} \alpha_{\chi}^{(j)} x_{\chi} & =S_{\lambda_{n}}\left(y_{j}\right)=y_{n} \\
& =\sum_{\chi<\nu} \alpha_{\chi}^{(n)} x_{\chi}, \quad(n=1,2, \ldots, j ; j \in N)
\end{aligned}
$$

where

$$
y_{j}=\sum_{\chi<\nu} \alpha_{\chi}^{(j)} x_{\chi}, \quad(j \in N)
$$

This gives

Thus

$$
\alpha_{\chi}^{(n)}= \begin{cases}\alpha_{\chi}^{(j)} ; & \chi<\lambda_{n} \\ 0 ; & \chi \geq \lambda_{n}\end{cases}
$$

$$
y_{j}=\sum_{n=1}^{j} \sum_{\lambda_{n-1} \leq \chi<\lambda_{n}} \alpha_{\chi}^{(n)} x_{\chi}, \quad\left(j \in N ; \lambda_{0}=1\right) .
$$

Put $\alpha_{\chi}=\alpha_{\chi}^{(n)}\left(\lambda_{n-1} \leq \chi<\lambda_{n} ; n \in N, \lambda_{0}=1\right)$. Then

$$
y_{j}=\sum_{n=1}^{j} \sum_{\lambda_{n-1} \leq \chi<\lambda_{n}} \alpha_{\chi} x_{\chi}, \quad(j \in N)
$$


Hence

$$
\lim _{j \rightarrow \infty} y_{j}=\sum_{n=1}^{\infty} \sum_{\lambda_{n}-1 \leq \chi<\lambda_{n}} \alpha_{\chi} x_{\chi}=\sum_{\chi<\nu} \alpha_{\chi} x_{\chi} .
$$

On the other hand $\left\{x_{\lambda}\right\}_{\lambda<\nu}$ does not satisfy property (I) since, for $\beta_{1}=\beta_{2}=1$ and $\beta_{\lambda}=0, \lambda<\nu, \lambda \neq 1,2$; we have $\left\|x_{1}\right\|=1$ and $\left\|x_{2}+\sum_{3 \leq \chi<\nu} 0 \cdot x_{\chi}\right\|=1$. But

$$
\left\|x_{1}+\left(x_{2}+\sum_{3 \leq \chi<\nu} 0 \cdot x_{\chi}\right)\right\|=\left\|e_{2}\right\|=1 .
$$

Finally the authors express their sincere thanks to the referee for his critical observations and suggestions towards the improvement of the paper.

\section{References}

[1] Bessa.ga. C., "Topological equivalence of unseparable reflexive Banach spaces", Ordinal resolutions of identity and monotone bases. Bull. Acad. Polon. Sci. 15(1967), 397-399.

[2] —_, "Topological equivalence of non-separable reflexive Banach spaces", Ordinal resolutions of identity and monotone bases. Proc. Sympos. on "Infinite dimensional topology" held in Baton Rouge, Louisiana, 1967, p.3-14, Princeton : Ann. of Math. Studies 69, Princeton Univ. Press (1972).

[3] Bessaga. C. and Pelczynski. A., Selected topics in infinite dimensional topology. Monografje Matematyczne, Warszawa (1975).

[4] Casazza. P., On a geometric condition related to boundedly complete bases and normal structure in Banach. spaces. Proc. Amer. Math. Soc. 36(1972), 443-447.

[5] Dorembus. L., Transfinite bases of subspaces in Hausdorff linear topological spaces. Math. Ann. 192(1971), 71-82.

[6] Jain, P.K. and Ahmad. K: A note on transfinite basis in Banach spaces. Tamkang Jour. Math. (To appear).

[7] Singer. I., Bases in Banach spaces-II. Springer-Verlag, Berlin (1981).

Department of Mathematics University of Delhi, Delhi-110007, INDIA. 